\title{
2011 Ostrowski Prize Awarded
}

IB MADSEN of the University of Copenhagen, KANNAN SOUNDARARAJAN of Stanford University, and DAVID PREISS of the University of Warwick have been awarded the 2011 Ostrowski Prize, which recognizes outstanding achievement in pure mathematics or in the foundations of numerical mathematics. The prize carries a monetary award of 75,000 Swiss francs (approximately US\$78,000).

\section{Citation for Ib Madsen}

Ib Madsen received his Ph.D. from the University of Chicago in 1970 . He was a member of the faculty at

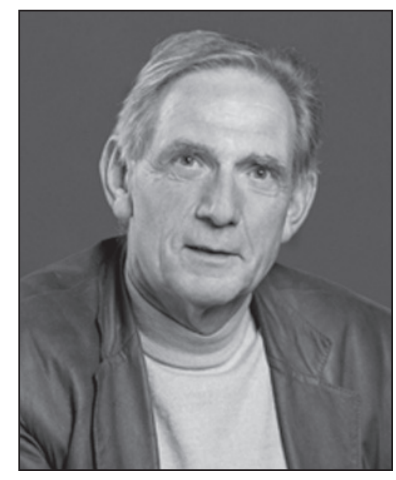

Ib Madsen the University of Aarhus from 1971 through 2008 and was very influential in building a strong topology group there. He has been a professor at the University of Copenhagen since 2008. He has held visiting positions at the University of Chicago, Stanford University, and Princeton University. He has been an invited speaker at the 1978 International Congress of Mathematicians (ICM) in Helsinki and a plenary speaker at the 2006 ICM in Madrid. He was chair of the topology panel for speaker selection to the 2002 ICM in Beijing, and he served as managing editor of Acta Mathematica from 1988 to 2000 . He is a member of the Royal Danish Academy of Sciences and Letters, the Royal Swedish Academy of Sciences, and the Royal Norwegian Academy.

Madsen has been instrumental in developing topological cyclic homology theory. This theory was developed as a tool for understanding Waldhausen's universal space $A(X)$, the only known way to approach the homotopy theory of diffeomorphism groups of high-dimensional manifolds. Topological cyclic theory is also, at present, the only known way to approach algebraic $K$-theory of nonsmooth rings and varieties. It is also the main tool for understanding the $K$-theory of symmetric ring spectra. Madsen has also accomplished breakthrough work on the stable moduli space of

DOI: http://dx.doi.org/10.1090/noti889
Riemann surfaces. The prize citation states, "Ib Madsen is an enormously significant figure in the world of mathematics and through his research and leadership has made a huge impact on the fields of geometry and topology."

\section{Citation for Kannan Soundararajan}

Kannan Soundararajan received his Ph.D. from Princeton University in 1998, has taught at the University of Michigan, and is currently a pro-

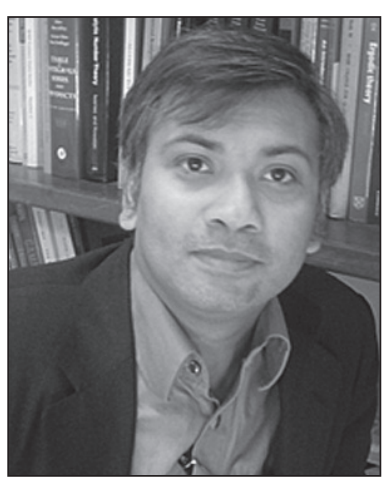

Kannan Soundararajan fessor at Stanford University. He has done groundbreaking work in number theory and analysis. In 2003 he was awarded the Salem Prize, in 2005 the SASTRA Ramanujan Prize, and in 2011 the Infosys Prize in Mathematical Sciences. He was an invited speaker at the 2010 ICM in Hyderabad, India.

S o u n d a r a r a jan's work has involved the quantum unique ergodicity conjecture of Rudnick and Sarnak; the behavior of $L$-functions within the critical strip; and pretentious characters, jointly with A. Granville. Also with Granville, he established an uncertainty principle for multiplicative functions that vastly extends the results of Maier on irregularities of distribution in the primes. Together with Konyagin, he improved the best known estimates for the number of solutions, in terms of the cardinality of a set of primes $S$, of the $S$-unit equation $a+b=c$ with $a, b$, and $c$ coprime integers, all of whose prime factors are from $S$. He has also given "the sharpest results to date, subject to the Riemann hypothesis, on partial sums of the Möbius function." The prize citation says "Soundararajan has produced a cornucopia of fundamental results in the last five years to go along with his brilliant earlier work."

\section{Citation for David Preiss}

David Preiss received his undergraduate and advanced degrees from Charles University in Prague, Czechoslovakia. In 1990 he became Astor Professor of Mathematics at University College in 
London. He has been at the University of Warwick since 2006. He is a Fellow of the Royal Society and received the London Mathematical Society's Pólya Prize in 2008.

According to the prize citation, "Preiss is undoubtedly the leading researcher in the world in geometric measure theory, where his major achievement was solving the density problem that

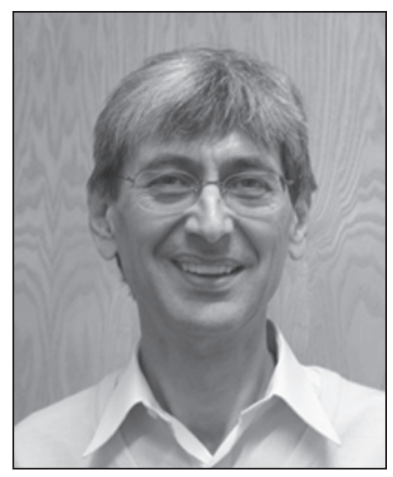

David Preiss had been stimulating the development of the theory since the time it was founded by Besicovitch and Federer." His early work in real analysis and descriptive set theory includes the positive solution of a problem of Klee. In functional analysis he is best known for his breakthrough result that every Lipschitz function on a Banach space with separable dual is Fréchet differentiable on a dense set. In recent years his research has focused on developing ideas initiated in his proof of Fréchet differentiability of Lipschitz functions. He reached a breakthrough by showing the existence of points of Fréchet differentiability of complex-valued Lipschitz functions on real Hilbert spaces. Jointly with G. Alberti and M. Csörnyei, he discovered new classes of exceptional sets that are described by the possibility of decomposing them into sets negligible on many curves.

\section{About the Prize}

The Ostrowski Foundation was created by Alexander Ostrowski, for many years a professor at the University of Basel. He left his entire estate to the foundation and stipulated that the income should provide a prize for outstanding recent achievements in pure mathematics and the foundations of numerical mathematics. The prize is awarded every other year. The prize jury consists of representatives from the universities of Basel, Jerusalem, and Waterloo and from the academies of Denmark and the Netherlands. Previous recipients of the Ostrowski Prize are Louis de Branges (1989), Jean Bourgain (1991), Miklos Laczkovich (1993), Marina Ratner (1993), Andrew Wiles (1995), Yuri Nesterenko (1997), Gilles Pisier (1997), Alexander Beilinson (1999), Helmut Hofer (1999), Henryk Iwaniec (2001), Peter Sarnak (2001), Richard L. Taylor (2001), Paul D. Seymour (2003), Ben Green (2005), Terence Tao (2005), Oded Schramm (2007), and Sorin Popa (2009).

-Elaine Kehoe

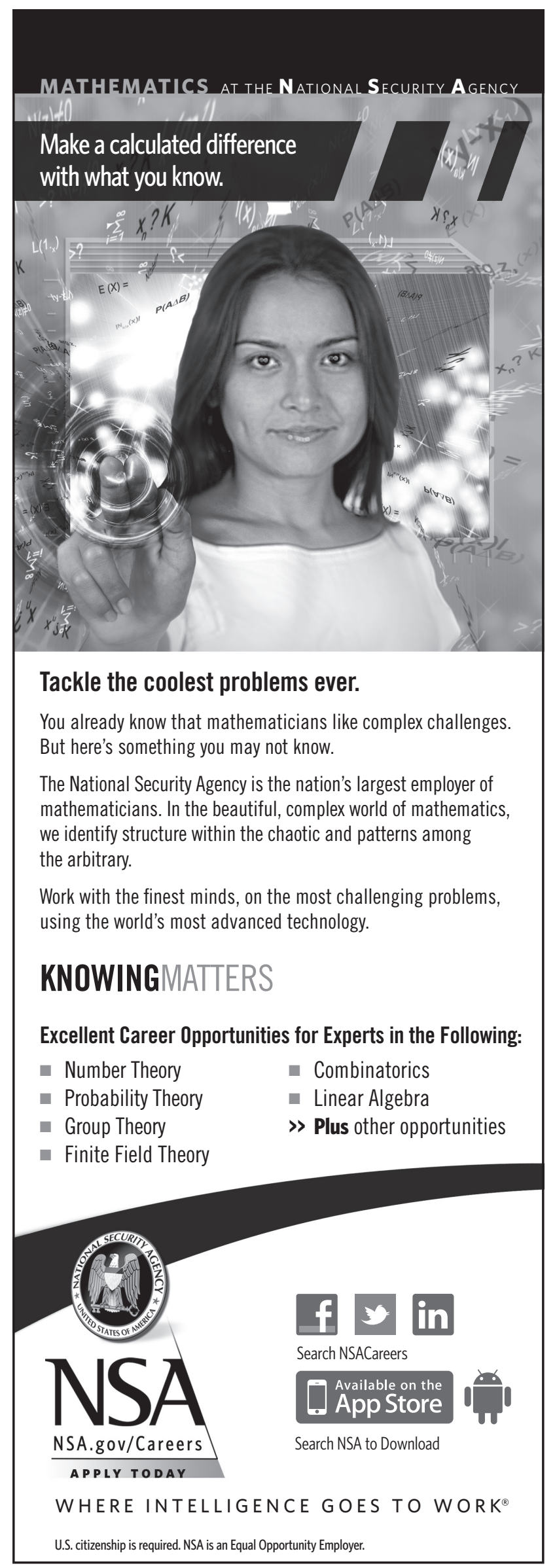

You already know that mathematicians like complex challenges. But here's something you may not know.

The National Security Agency is the nation's largest employer of mathematicians. In the beautiful, complex world of mathematics, we identify structure within the chaotic and patterns among the arbitrary.

Work with the finest minds, on the most challenging problems, wsing the world's most advanced technology.

\section{KNOWINGMATTERS}

Excellent Career Opportunities for Experts in the Following:

Number Theory

Probability Theory

Group Theory

》> Plus other opportunities

- Finite Field Theory 